\title{
Urine measurement indicates the plasma brain natriuretic peptide concentration during optimization of heart failure treatment
}

\author{
Robert G. Hahn, Tiny Jaarsma, Nana Waldréus and Gerard C. M. Linssen
}

\section{Linköping University Post Print}

\section{Tweet}

N.B.: When citing this work, cite the original article.

This is an electronic version of an article published in:

Robert G. Hahn, Tiny Jaarsma, Nana Waldréus and Gerard C. M. Linssen, Urine measurement indicates the plasma brain natriuretic peptide concentration during optimization of heart failure treatment, 2016, Scandinavian Journal of Clinical and Laboratory Investigation, (76), 2, 112 117.

Scandinavian Journal of Clinical and Laboratory Investigation is available online at informaworldTM:

http://dx.doi.org/10.3109/00365513.2015.1108454

Copyright: Taylor \& Francis: STM, Behavioural Science and Public Health Titles http://www.tandf.co.uk/journals/default.asp

Postprint available at: Linköping University Electronic Press http://urn.kb.se/resolve?urn=urn:nbn:se:liu:diva-126841 
Urine measurement indicates the plasma brain natriuretic peptide concentration during optimization of heart failure treatment

Robert G. Hahn ${ }^{1}$, Tiny Jaarsma ${ }^{2}$, Nana Waldréus ${ }^{1,2}$, Gerard C. M. Linssen ${ }^{4}$

${ }^{1}$ Department of Research, Södertälje Sjukhus, Södertälje, Sweden;

${ }^{2}$ Department of Social and Welfare Studies, Faculty of Health Sciences, Linköping University, Linköping, Sweden; ${ }^{4}$ Department of Cardiology, Hospital Group Twente, Almelo, the Netherlands

\section{Corresponding author:}

Robert G. Hahn, MD, PhD

Professor of Anaesthesiology and Intensive Care

Research Unit, Södertälje Hospital

15286 Södertälje, Sweden

Telephone: +467395660972

E-mails: r.hahn@ elia.com

Robert.hahn@sodertaljesjukhus.se 


\begin{abstract}
Aim: To assess the correlation between the amino-terminal pro-hormone brain natriuretic peptide (NT-proBNP) concentration in blood and urine during a period when actively adjusting the treatment of heart failure (HF).
\end{abstract}

Methods: Plasma and urine analyses of NT-proBNP were compared in 51 patients on admission to and discharge from a nurse-led outpatient clinic where HF treatment was optimized. The median time between the two measurements was 42 days. Correlations were analyzed using linear regression, where $\mathrm{R}^{2}$ is the degree of variability in the plasma NT-proBNP concentration that can be accounted for by the urinary NT-proBNP.

Results: There was a statistically significant linear relationship between the urine and plasma concentrations of NT-proBNP on both occasions, but $\mathrm{R}^{2}$ varied greatly depending on how the data were presented. The correlation between the raw data showed an $\mathrm{R}^{2}$ of only $30 \%$, and it almost doubled upon logarithm transformation, which shows that the variability (error) was concentration-dependent. Correction of the urinary NT-proBNP for urinary creatinine further increased $\mathrm{R}^{2}$ for the logarithm-transformed correlation to $68 \%$ on admission and $76 \%$ on discharge. The highest $\mathrm{R}^{2}(77 \%)$ was obtained when the relative changes in urinary NT-proBNP/creatinine between admission and discharge were compared with the corresponding relative changes in the plasma concentration. The sensitivity and specificity of the urine in indicating plasma concentration changes $>10 \%$ were $82 \%$ and $86 \%$, respectively.

Conclusion: Relative changes in plasma NT-proBNP could be reliably estimated from urine samples during a period of optimization of HF treatment.

Keywords: heart failure; urinary NT-proBNP; natriuretic peptide, brain; patient monitoring 


\section{Introduction}

Heart failure (HF) is a leading cause of hospitalization for elderly patients worldwide. Approximately 1-2\% of the adult population has HF, but the prevalence is $\geq 10 \%$ among persons being 70 years of age or older [1]. The condition is associated with fatigue, thirst, shortness of breath, latent or manifest pulmonary edema, and a worse prognosis [3]. Treatment primarily includes diuretic drugs, beta-blockers, angiotensinconverting enzyme (ACE) inhibitors, fluid and salt restriction, and lifestyle changes.

Changes in the amino-terminal pro-hormone brain natriuretic peptide (NTproBNP) level during in-hospital treatment are strong predictors of mortality and early readmission [2-4]; therefore, NT-proBNP levels might be successfully used to guide the treatment of HF patients. An increase in NT-proBNP concentration indicates a worsening of the condition and, together with worsening symptoms, may call for a period of hospitalization or intensified treatment in the patient's home [1-4].

Monitoring of HF with NT-proBNP sampling requires one or several visits to a hospital or a general practitioner, which, among frail, immobile older patients, might be an exhausting and complicated undertaking. Measuring NT-proBNP at home is feasible [4], but it is complicated by the fact that BNP requires blood sampling. Urinary analysis of NTproBNP could be performed more easily, and recent studies show that NT-proBNP may be measured not only in the blood but also in the urine [5-9].

The aim of the present study was to analyze the correlation between NT-proBNP measurements in blood and urine during a period of active adjustment of HF treatment. The hypothesis was that trends in the NT-proBNP levels during treatment of the HF condition can be indicated correctly by using urine instead of blood analysis. 


\section{Methods}

\section{Study design}

A prospective observational study was performed at two hospitals with nurse-led HF clinics in Stockholm, Sweden (Söder Hospital and Karolinska University Hospital at Huddinge). The nurses specialize in managing patients with $\mathrm{HF}$ and are educated to make protocol-guided changes in their medication.

The local ethics committee in Stockholm approved the study (dnr. 2012/42-32), which complied with the Declaration of Helsinki and was part of a larger evaluation of thirst in HF. All patients were informed about the study verbally and in writing before giving their consent.

\section{Patients}

Patients were recruited from those referred to the nurse-led HF outpatient clinics from a cardiology department after being hospitalized for HF, or occasionally, after being treated in the outpatient setting for the worsening of HF. The inclusion criteria were unstable HF (as evidenced by increasing signs and symptoms of HF for $\geq 1$ month) with a New York Heart Association (NYHA) functional class of II-IV, and aged $\geq 60$ years. All patients had a left ventricular ejection fraction (LVEF) of $\leq 50 \%$ as determined by transthoracic echocardiography shortly before optimizing the HF treatment. Exclusion criteria were insulin-treated diabetes, significant psychiatric illness, dementia, advanced kidney disease, palliative end-of-life care, pulmonary disease treated with oxygen, and inability to communicate in Swedish.

Patients were enrolled in the study and baseline data were collected on admission to the HF clinic. The second set of data was collected at the time of outpatient discharge, when the patients were considered to be optimized with respect to their HF treatment. The time period between admission and discharge varied between 13 and 169 days (median: 42).

\section{Measurements}

The following clinical data were collected: LVEF, heart rate, arterial pressure, body weight, body mass index, NYHA functional class, co-morbidities, and medications. 
At the start and end of care at the HF clinic, the NT-proBNP concentration in the patients' plasma and urine were measured using an Elecsys analyser (Elecsys proBNP, Roche Diagnostics, Mannhein, Germany) with a coefficient of variation of $1.2-1.5 \%$ for plasma and $4.4-5.0 \%$ for urine. This equipment has previously been found suitable for measuring urinary NT-proBNP levels [8]. Samples were immediately sent for analysis, without being frozen, to the certified clinical chemistry laboratory of the Karolinska University Hospital in Stockholm. The results were expressed in $\mathrm{ng} / \mathrm{L}$ (which should be divided by 8.46 to be converted into $\mathrm{pmol} / \mathrm{L})$.

\section{Statistics}

The data were expressed as means and standard deviations (SD); values showing a skewed distribution were presented as the median (25th-75th percentiles). Relationships between the parameters were evaluated using linear regression analysis, where skewed data were logarithm-transformed. Here, the fraction of the variability of $y$ that could be explained by $\mathrm{x}$ is given by the coefficient of determination $\mathrm{R}^{2}$ (squared correlation coefficient). $P<0.05$ was considered as statistically significant. 


\section{Results}

\section{Patients}

Sixty-nine patients were asked to participate, all of whom responded favorably. Three of them were unable to complete the study due to acute clinical deterioration. Complete laboratory data sets were missing from another 15 patients, leaving 51 for the final analysis. The data on demographics, morbidity, and medical treatments are shown in Table I.

NT-proBNP

The plasma concentration of NT-proBNP on admission was 1,900 (992-2,823 ng/L) ng/L, and the corresponding urinary NT-proBNP was 83 (44-194) ng/L.

The median values for the group hardly changed at all between admission and discharge, whereas changes in some individual patients were large: $27 \%$ of the patients had a change of $>50 \%$ in their plasma concentration. The corresponding fraction for the urinary concentration was $30 \%$.

\section{Correlations between plasma and urine data}

The relationship between the plasma and urinary measurements of NT-proBNP on admission and discharge was statistically significant $(P<0.001)$. Between $28 \%$ and $30 \%$ of the variability in the plasma NT-proBNP could be explained (i.e., $\mathrm{R}^{2}$ ) by the urinary NT-proBNP concentration (Fig. 1A, D).

The logarithm transformation of the data increased this fraction to $49 \%$ on admission and to $58 \%$ on discharge (Fig. $1 \mathrm{~B}$ and E).

Further strengthening of the correlation between the plasma and urine data was obtained when accounting for dilution by dividing the urinary NT-proBNP concentration by urinary creatinine: $68 \%$ and $76 \%$, respectively, of the variability in the ${ }^{10} \log$ urinary NT-proBNP/creatinine could then be explained by the ${ }^{10} \log$ plasma NT-proBNP (Fig. 1C and $\mathrm{F}$ ). In contrast, $\mathrm{R}^{2}$ was only $33 \%$ on admission and $38 \%$ on discharge when the data were not logarithm-transformed. 
The relative changes in the raw data on the plasma and urinary NT-proBNP correlated poorly, with $\mathrm{R}^{2}$ being only $12 \%(P<0.01$; data not shown). However, correcting for the creatinine excretion increased $\mathrm{R}^{2}$ to $77 \%$ (Fig. 2). Increasing plasma values were correctly indicated by the urine sample in $76 \%$ of the cases (sensitivity) and a reduction of the plasma NT-proBNP in $85 \%$ of the patients (specificity).

The sensitivity and specificity of the urine samples to detect the direction of any plasma change rose to $82 \%$ and $86 \%$, respectively, when disregarding changes in plasma NT-proBNP within $\pm 10 \%$. To disregard minor changes appeared to be relevant, as the relative changes of two parameters include four measurements (the two urinary concentrations of which were obtained with a coefficient of variation of almost 5\%).

The agreement between the relative changes in plasma NT-proBNP and the creatinine-corrected urinary NT-proBNP is shown in Fig. 3.

\section{Co-variates}

Overall, the plasma NT-proBNP was higher in the higher NYHA classes (KruskalWallis test; $P<0.003$ ); the median values for NYHA classes I, II, and III were $219 \mathrm{ng} / \mathrm{L}$, $1490 \mathrm{ng} / \mathrm{L}$, and $2510 \mathrm{ng} / \mathrm{L}$, respectively. The corresponding urinary NT-proBNP levels were $16 \mathrm{ng} / \mathrm{L}, 90 \mathrm{ng} / \mathrm{L}$, and $113 \mathrm{ng} / \mathrm{L}$ for NYHA classes I, II, and III, respectively $(P<0.01$; four patients were classified as NYHA I on discharge).

The plasma NT-proBNP concentration and the urinary NT-proBNP/creatinine ratio tended to increase with serum creatinine. $\mathrm{R}^{2}$ for these correlations was $14 \%$ and $18 \%$ (both $\mathrm{P}<0.001$ ), respectively.

Adding any one of the other parameters (age, body weight, serum creatinine, atrial fibrillation, NYHA class, or left ventricular ejection fraction (stepwise multiple regression analysis)) did not significantly strengthen the correlation shown in Fig. 2 (i.e., the one between the relative changes in plasma NT-proBNP and the creatinine-corrected urinary NT-proBNP). 


\section{Discussion}

\section{Findings}

Our results confirm that the NT-proBNP can be measured in urine as well as in plasma in patients with HF [5-11]. The urinary concentrations were 5-10\% of those measured in plasma, and the degree of correlation between the crude values was found to be quite low. The apparent reduction of the variability upon logarithm transformation of the data suggests that the error is concentration-dependent. A stronger correlation between plasma and urine NT-proBNP concentrations was also obtained on correction for urine flow by considering the urinary creatinine concentration, but the considerable deviations with higher plasma levels suggests that the logarithm transformation should be retained.

A useful application of the urinary NT-proBNP measurements appeared to be when changes in individual patients over time were depicted. After correcting the urinary NT-proBNP for creatinine, a strong correlation was found between the relative changes in this ratio and the plasma concentration of NT-proBNP (Fig. 2). Almost four-fifths of the variability in the change in plasma NT-proBNP could then be accounted for by urinary NT-proBNP/creatinine.

The fact that the correlation between these parameters was quite poor when analyzed separately on admission and discharge suggests that a patient-specific renal clearance constant determines the size of the fraction of the plasma NT-proBNP that will be reflected in the urine samples. This renal clearance is relatively stable from sample to sample, as judged from the high precision by which relative changes in plasma NTproBNP could be predicted from the urinary ratio of NT-proBNP/creatinine.

\section{Previous studies}

$\mathrm{Ng}$ et al. pointed out the possibility of using urine analysis of natriuretic peptides to diagnose HF a decade ago [5]. They compared 34 NYHA class IV patients with 82 normal subjects and found that the HF patients showed dramatic elevations of the peptide concentrations in both body fluids. $\mathrm{R}^{2}$ for the correlation between these parameters was about $40 \%$ when all of the data were logarithm-transformed.

Cortés et al. reported that urinary NT-proBNP can be used to diagnose HF with a high degree of confidence [6]. They also found higher urinary concentrations for the 
higher NYHA classes and lower concentrations in obese patients, which the present study confirms. Overall $\mathrm{R}^{2}$ for the linear plasma/urine relationship was again about $40 \%$ for the HF patients. A later study showed that both plasma and urinary NT-proBNP levels remained stable in clinically stable HF patients over a two-year period [7].

Jungbauer et al. reported that $\mathrm{R}^{2}$ was $62 \%$ for the correlation between plasma and urinary NT-proBNP. They also confirmed that urinary concentration increases with the NYHA class; the cut-off for the diagnosis of HF was set as $14 \mathrm{ng} / \mathrm{L}$ [8].

The renal excretion of NT-proBNP is unaffected by kidney dysfunction [9] and moderate exercise [10], but it is reduced by poor renal plasma flow, which is common in HF patients [11]. Linssen et al. collected urine over a 24-hour period instead of using spot samples and found that the urinary concentration and diurnal excretion of NT-proBNP were lower in HF patients than in healthy control subjects. There was an inverse correlation between the plasma concentration and urinary excretion of NT-proBNP [11], which differs from other work [5-7]. However, Cortez et al. also found that the NTproBNP concentrations are higher in the blood of HF patients but not in healthy controls [6]. Therefore, current evidence suggests that the renal clearance of NT-proBNP is greatly reduced in $\mathrm{HF}$ but that the remarkable elevation $(\approx 50$-fold $)$ of the plasma concentration still results in urinary concentrations that are still proportional to the plasma level.

More than half of our patients had atrial fibrillation, which is known to frequently coexist with HF. Atrial fibrillation increases the plasma NT-proBNP by approximately $50 \%[12,13]$, although we found no statistically significant influence on the correlations. This is probably because atrial fibrillation does not alter the relationship between NTproBNP in plasma and urine.

\section{Limitations}

Previous work focused on the use of urine sampling, alone or combination with blood analysis, as a diagnostic tool to distinguish between those who have HF from those who do not. The present work had a more limited scope, but it is the first to assess whether relative changes in urinary NT-proBNP can be used to indicate changes in plasma concentrations during a period of the active optimization of HF treatment.

Our urinary NT-proBNP concentrations clearly needed correction for creatinine excretion to reduce inter-subject variability, which is a common procedure to account for sample dilution. Researchers who have not found this correction to be necessary have 
sampled urine early in the morning or several hours after breakfast, which ensures less inter-subject variability in urine flow [5,6]. In contrast, we sampled throughout the day whenever the patient was scheduled for a visit to the HF clinic, which means that the time relationship between sampling and food and fluid intake was not standardized.

Data on HF symptoms on admission and discharge were collected, but they were not compared with the NT-proBNP changes in this report.

\section{Implications}

Substantial changes in plasma NT-proBNP concentration correspond to changes in risk for worsening or improving HF symptoms and prognosis. Rapidly increasing NTproBNP levels may be an early warning sign before acute cardiac decompensation develops; therefore, NT-proBNP analysis is a widely accepted tool for monitoring HF [1].

Care of HF patients would significantly improve by implementing frequent NTproBNP monitoring in the home care setting and in increased telemonitoring of HF patients [14]. A research project aimed to implement BNP monitoring in the home (the HABIT trial) showed that patients can be taught to take their own blood for testing [4]. The present finding of a stable relationship between relative changes in plasma and urinary concentrations of NT-proBNP over a period of HF treatment optimization opens the possibility of using urine analysis to monitor HF in the home care setting. Patients' adherence to participating in such a program would probably be high if essential information about NT-proBNP changes could be obtained by urine sampling.

The use of a home care system is likely to improve patients' feelings of security and to decrease their symptom burden if combined with easier (online) access to medical guidance regarding the delicate balance between fluid intake and medication. Such a program could hopefully improve their quality of life and decrease the incidence of acute hospital admissions, which constitutes $70 \%$ of the healthcare costs associated with HF [15].

\section{Conclusion}

Changes in the plasma concentration of NT-proBNP can be monitored by analyzing urine samples with a high degree of confidence. Serial measurements of urinary NT-proBNP more accurately predicted plasma concentrations of NT-proBNP than single measurements. This is probably due to inter-subject variation in the renal clearance of NT- 
proBNP, which cancels out when changes are studied. Further studies are needed to validate the practical aspects, the medical and psychological benefits and the risks involved in allowing patients to monitor their own HF condition by measuring the creatinine-corrected urinary NT-proBNP in the home care setting. 
Table I. Patient characteristics, co-morbidities, and medical treatment.

\begin{tabular}{|c|c|c|}
\hline & Admission & Discharge \\
\hline $\mathbf{N}$ & 51 & 51 \\
\hline Age (years) & $72(7)$ & \\
\hline Males (N, \%) & $42(83 \%)$ & \\
\hline Body weight (kg) & $83.8(17.8)$ & $83.2(17.3)$ \\
\hline Body mass index $\left(\mathrm{kg} / \mathrm{m}^{2}\right)$ & $27.2(5.2)$ & $27.0(5.0)$ \\
\hline LVEF (\%) & $33(10)$ & \\
\hline Heart failure of new onset $(\mathrm{N}, \%)$ & $25(51 \%)$ & \\
\hline NYHA class I / II / III / IV (N) & $0 / 29 / 22 / 0$ & $4 / 30 / 17 / 0$ \\
\hline \multicolumn{3}{|l|}{ Arterial pressure (mmHg) } \\
\hline Systolic & $123(19)$ & $120(20)$ \\
\hline Diastolic & $75(12)$ & $73(12)$ \\
\hline Plasma creatinine $(\mu \mathrm{mol} / \mathrm{L})$ & $103(31)$ & $103(30)$ \\
\hline \multicolumn{3}{|l|}{ Co-morbidities (N, \%) } \\
\hline Atrial fibrillation & $30(59 \%)$ & \\
\hline Hypertension & $20(39 \%)$ & \\
\hline Myocardial infarction & $10(20 \%)$ & \\
\hline Valvular disease & $10(20 \%)$ & \\
\hline Diabetes & $9(18 \%)$ & \\
\hline Malignancy & $7(14 \%)$ & \\
\hline Pulmonary disease & $7(14 \%)$ & \\
\hline \multicolumn{3}{|l|}{ Medication (N, \%) } \\
\hline Beta-blockers & $50(98 \%)$ & $49(96 \%)$ \\
\hline ACE inhibitors & $37(73 \%)$ & $38(75 \%)$ \\
\hline Aldosterone antagonists & $8(16 \%)$ & $12(24 \%)$ \\
\hline Diuretics & $37(73 \%)$ & $35(69 \%)$ \\
\hline Angiotensin II receptor antagonists & $8(16 \%)$ & $13(25 \%)$ \\
\hline Fluid restriction $(\mathrm{N}, \%)$ & $24(47 \%)$ & $20(39 \%)$ \\
\hline
\end{tabular}

Continuous data are given as means (SD).

Left ventricular ejection fraction (LVEF); New York Heart Association (NYHA); angiotensin-converting enzyme (ACE). 


\section{References}

[1] McMurray JJ, Adamopoulus S, Anker SD, Auricchio A, Böhm M, Dickstein K, Falk V, Filippatos G, Fonseca C, Gomez-Sanchez MA, Jaarsma T, Köber L, Lip GY, Maggioni AP, Parkhomenko A, Pieske BM, Popescu BA, Rönnevik PK, Rutten FH, Schwitter J, Seferovic P, Stepinska J, Trindade PT, Voors AA, Zannad F, Zeiher A. ESC guidelines for the diagnosis and treatment of acute and chronic heart failure 2012: the Task Force for the diagnosis and treatment of acute and chronic heart failure 2012 of the European Society of Cardiology. Developed in collaboration with the Heart Failure Association of the ESC (CHFA) and endorsed by the European Society of Intensive Care Medicine (ESICM). Eur J Heart Fail 2012;14:803-69.

[2] Cheng V, Kazanagra R, Garcia A, Lenert L, Krishnaswamy P, Gardetto N. A rapid bedside test for B-type peptide predicts treatment outcomes in patients admitted for decompensated heart failure: a pilot study. J Am Coll Cardiol 2001;37:386-91.

[3] van Veldhuisen DJ, Linssen GCM, Jaarsma T, van Gilst WH, Hoes AW, Tijssen JGP,Paulus WJ, Voors AA, Hillege HL. B-type natriuretic peptide and prognosis in heart failure patients with preserved and reduced ejection fraction. J Am Coll Cardiol 2013;61:1498-1506.

[4] Maisel A, Barnard D, Jaski B, Frivold G, Marais J, Azer M, Miyamoto MI, Lombardo D, Kelsay D, Borden K, Iqbal N, Taub PR, Kupfer K, Clopton P, Greenberg B. Primary results of the HABIT trial (heart failure assessment with BNP in the home). J Am Colleg Cardiol 2013;61:1726-35.

[5] Ng LL, Geeranavar S, Jennings SC, Loke I, O’Brien J. Diagnosis of heart failure using urinary natriuretic peptides. Clin Sci 2004;106:129-33.

[6] Cortez R, Portoles M, Salvador A, Bertomeu V, de Burgos FG, Martinez-Dolz L, Lleti ER, Climent V, Jordán A, Payá R, Rivera M. Diagnostic and prognostic value of urine NT-proBNP levels in heart failure patients. Eur J Heart Fail 2006;8:621-7. 
[7] Cortez R, Rivera M, Salvador A, Bertomeu V, de Burgos FG, Roselló-Lleti E, Portolés M, Payá R, Martinez-Dolz L, Climent V. Variability of NT-proBNP plasma and urine levels in patients with stable heart failure: a 2-year follow-up study. Heart 2007;93:95762.

[8] Jungbauer CG, Buchner S, Birner C, Resch M, Heinicke N, Debl K, Buesing M, Biermeier D, Schmitz G, Riegger G, Luchner A. N-terminal pro-brain natriuretic peptide from fresh urine for the biochemical detection of heart failure and left ventricular dysfunction. Eur J Heart Fail 2010;12:331-7.

[9] Manzano-Fernández S, Januzzi JL, Boronat-García M, Pastor P, Albaladejo-Otón M, Garrido IP, Bayes-Genis A, Valdés M, Pascual-Figal DA. Impact of kidney dysfunction on plasma and urinary $\mathrm{N}$-terminal pro-B-type natriuretic peptide in patients with acute heart failure. Congest Heart Fail 2010;16:214-20.

[10] Schou M, Dalsgaard MK, Clemmesen O, Dawson EA, Yoshiga CC, Nielsen HB, Gustafsson F, Hildebrandt PR. Secher NH. Kidneys extract BNP and NT-proBNP in healthy young men. J Appl Physiol 2005;99:1676-80.

[11] Linssen GC, Damman K, Hillege HL, Navis G, van Veldhuisen DJ, Voors AA. Urinary $\mathrm{N}$-terminal prohormone brain natriuretic peptide excretion in patients with chronic heart failure. Circulation 2009;120:35-41.

[12] Shin DI, Jaekel K, Shley P, Sause A, Müller M, Fueth R, Scheffold T, Guelker H, Horlitz M. Plasma levels of NR-proBNP in patients with atrial fibrillation before and after electrical cardioversion. Z Kardiol 2005;94:795-800.

[13] Linssen GCM, Rienstra M, Jaarsma T, Voors AA, van Gelder IC, Hillege HL, van Veldhuisen DJ. Clinical and prognostic effects of atrial fibrillation in heart failure patients with reduced and preserved left ventricular ejection fraction. Eur J Heart Fail 2011;13:1111-20.

[14] Boyne JJ, Vrijhoef HJ, Spreeuwenberg M, De Weerd G, Kragten J, Gorgels AP; TEHAF Investigators. Effects of tailored telemonitoring on heart failure patients' knowledge, self-care, self-efficacy and adherence: a randomized controlled trial. Eur J Cardiovasc Nurs 2014;13:243-52.

[15] Stålhammar J, Stern L, Linder R, Parikh SSR, Ariely R, Wikström G. Resource utilization and cost of heart failure associated with reduced ejection fraction in Swedish patients. J Med Econ 2012;5:938-94. 

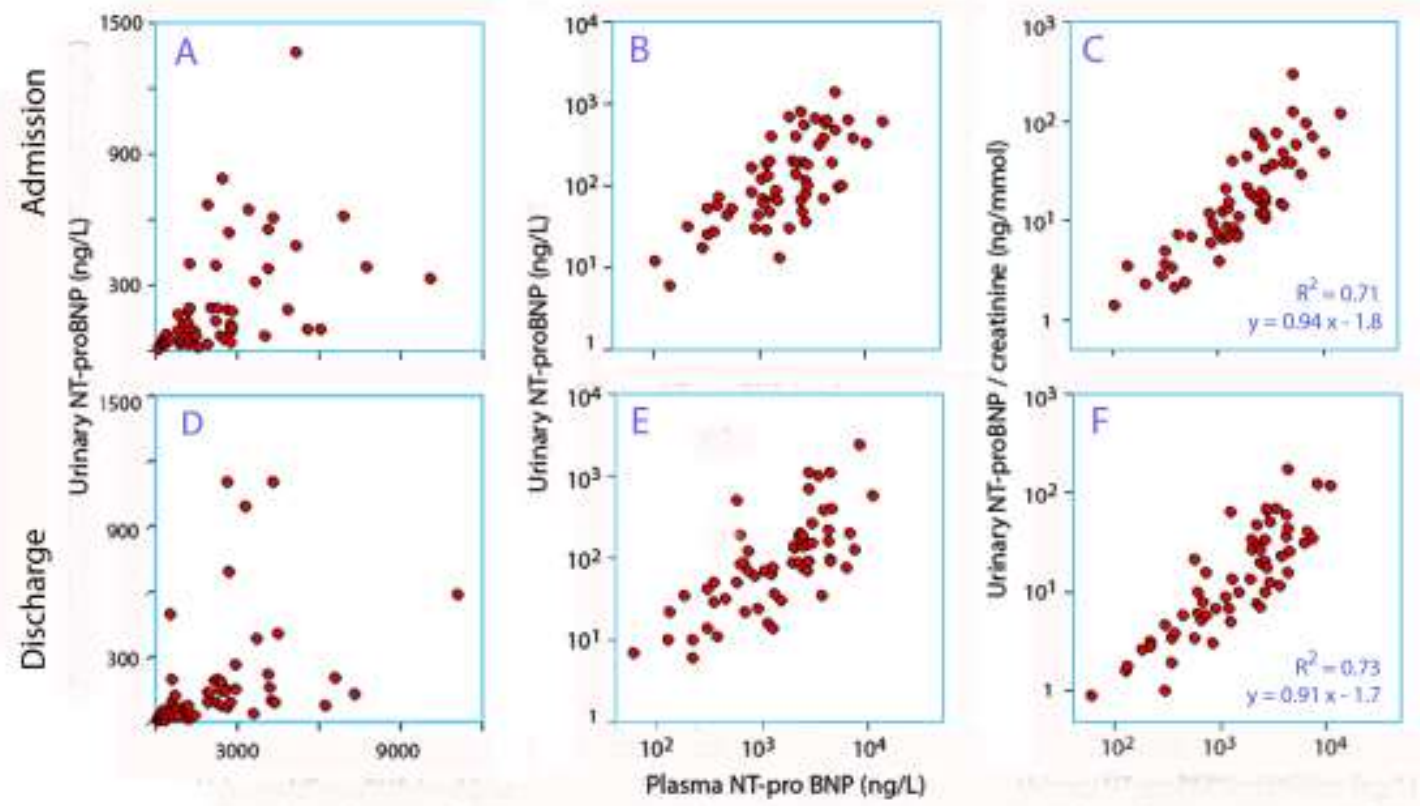

\section{Figure 1.}

Scatterplots of the association between plasma and urinary NT-proBNP concentrations in patients with HF on admission (upper row) and discharge (lower row). The data in subplots B, C, D and E are ${ }^{10} \log$-transformed. One extreme outlier is not shown in Subplot D. 


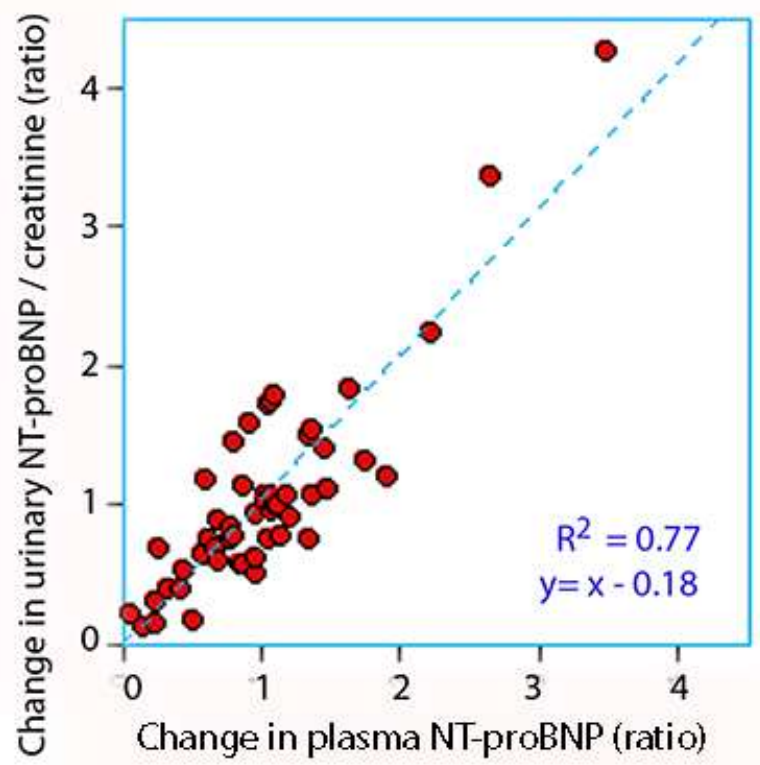

\section{Figure 2.}

Linear relationship between the relative changes in the plasma and creatinine-corrected urinary NT-proBNP concentrations between two visits to the hospital in patients with HF. 


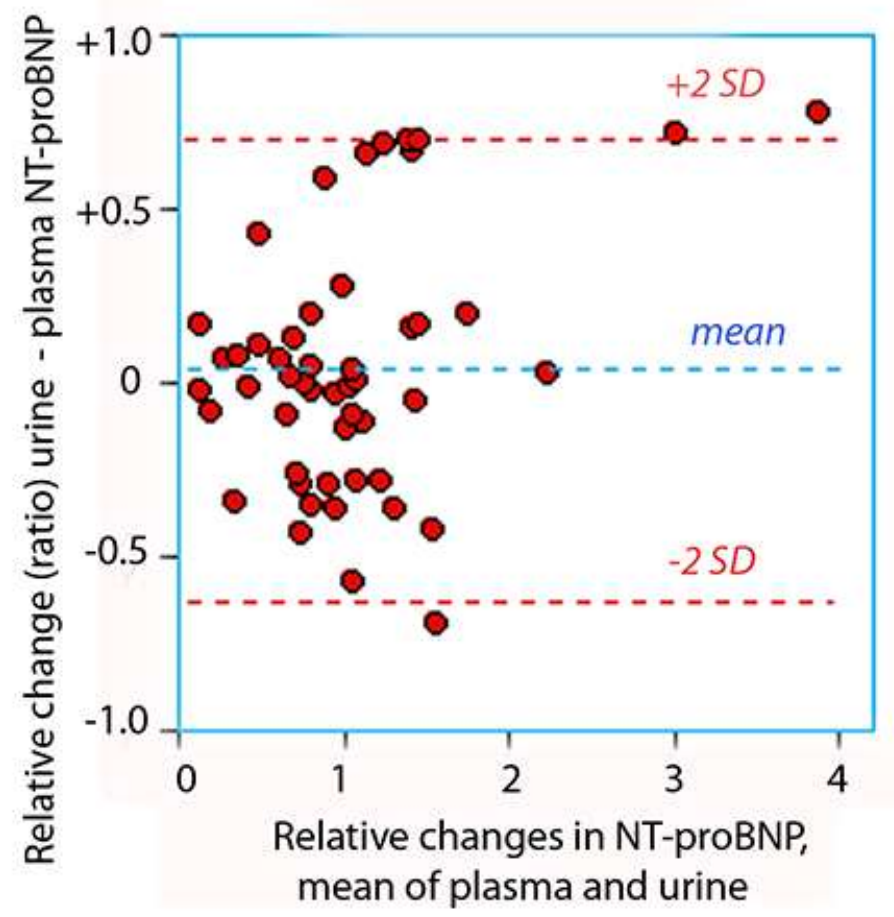

\section{Figure 3.}

Bland-Altman plot showing the agreement between the relative changes in the plasma and creatinine-corrected urinary NT-proBNP concentrations between two visits to the hospital in patients with HF (same data as in Figure 2). 